\title{
NEUTRINO FACTORY AND MUON COLLIDER R\&D
}

\author{
STEVE GEER \\ Fermi National Accelerator Laboratory, P.O. Box 500, Batavia, IL 60510, USA \\ E-mail: sgeer@fnal.gov
}

\begin{abstract}
European, Japanese, and US Neutrino Factory designs are presented. The main R\&D issues and associated R\&D programs, future prospects, and the additional issues that must be addressed to produce a viable Muon Collider design, are discussed.
\end{abstract}

\section{Introduction}

The development of a very intense muon source capable of producing a millimole of muons per year would enable a Neutrino Factory 1 , and perhaps eventually a Muon Collider 2 , to be built. In the last two years Neutrino Factory physics studies 3 have mapped out an exciting Neutrino Factory physics program. In addition, Neutrino Factory feasibility studies 4.5 have yielded designs that appear to be "realistic" provided the performance parameters for the critical components can be achieved. Some of the key components will need a vigorous $R \& D$ program to meet the requirements. Neutrino Factory R\&D activities in Europe 6, Japan 6, 8, and the US 9 are ongoing and have, in fact, resulted in three promising variants of the basic Neutrino Factory design. In the following the various Neutrino Factory schemes are briefly described. The main R\&D issues, and the ongoing $R \& D$ programs are summarized, and R\&D results presented. Finally, the additional issues that must be addressed before a Muon Collider can be proposed are briefly discussed.

\section{Neutrino Factory Schemes}

In all of the present Neutrino Factory schemes an intense multi-GeV proton source is used to make low energy charged pions. The pions are confined within a large acceptance decay channel. The daughter muons produced from $\pi^{ \pm}$decays are also confined within the channel, but they occupy a large phase-space volume, and this presents the main challenge in designing Neutrino Factories and Muon Colliders.

In the US and European designs the strategy is to first reduce the energy spread of the muons by manipulating the longitudinal phase-space they occupy using a technique called "phase rotation". The transverse phase-space occupied by the muons is then reduced using "ionization cooling 10 ". After phase rotation and ionization cooling the resulting muon phase space fits within the acceptance of a normal type of accelerator. In the Japanese scheme an alternative strategy is pursued, in which the large muon phase-space is accommodated using so called FFAG's, which are very large acceptance accelerators. Finally, in all three schemes the muons are accelerated to the desired final energy (typically in the range from $20-50 \mathrm{GeV}$ ), and injected into a storage ring with either two or three long straight sections. Muons decaying within the straight sections produce intense neutrino beams. If the straight section points downwards, the resulting beam is sufficiently intense to produce thousands of neutrino interactions per year in a reasonably sized detector on the other side of the Earth $₫$ !

\subsection{US Scheme}

In the last 18 months there have been two Neutrino Factory "Feasibility" Studies 45 in the US. Within these studies engineering de- 


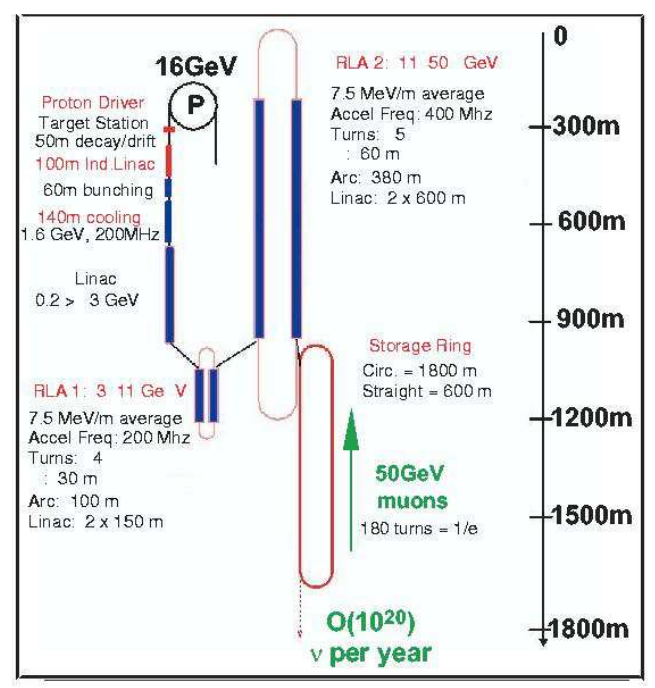

Figure 1. US Neutrino Factory schematic from design study I [4].

signs have been developed and detailed simulations performed for each piece of the Neutrino Factory complex. Study I was initiated by the Fermilab Director, and conducted between October 1999 and April 2000. The Study I design was for a $50 \mathrm{GeV}$ Neutrino Factory with the neutrino beams pointing down $13^{\circ}$ below the horizon, corresponding to a baseline of $2900 \mathrm{~km}$. A schematic is shown in Figure 1. The proton source for Study I 11 was assumed to be a $16 \mathrm{GeV}$ synchrotron producing $3 \mathrm{~ns}$ long bunches, operating at $15 \mathrm{~Hz}$, and providing a $1.2 \mathrm{MW}$ beam $\left(4.5 \times 10^{14}\right.$ protons per sec $)$ on an $80 \mathrm{~cm}$ long carbon target located within a $20 \mathrm{~T}$ solenoid. The solenoid radially confines essentially all of the produced $\pi^{ \pm}$. Downstream of the target and target solenoid the pions propagate down a $50 \mathrm{~m}$ long decay channel consisting of a $1.25 \mathrm{~T}$ super-conducting solenoid which confines the $\pi^{ \pm}$and their daughter muons within a warm bore of $60 \mathrm{~cm}$. At the end of the decay channel $95 \%$ of the initial $\pi^{ \pm}$ have decayed and, per incident proton, there are $\sim 0.2$ muons with energies $<500 \mathrm{MeV}$ captured within the beam transport system.
The muon system downstream of the decay channel is designed to produce a bunched cold muon beam with a central momentum of $200 \mathrm{MeV} / \mathrm{c}(\mathrm{T}=120 \mathrm{MeV})$. Beyond the decay channel. the first step is to reduce the muon energy spread using a $100 \mathrm{~m}$ long induction linac which accelerates the late low energy particles and de-accelerates the early high energy particles (phase rotation). After the induction linac, a $2.45 \mathrm{~m}$ long liquid hydrogen absorber is used to lower the central energy of the muons. Muons with energies close to the central value are then captured within bunches using a $201 \mathrm{MHz} \mathrm{RF}$ system within a $60 \mathrm{~m}$ long channel. Throughout the induction linac, liquid hydrogen absorber, and bunching system the muons are confined radially using a solenoid field of a few Tesla. The buncher produces a string of muon bunches, captured longitudinally, but still occupying a very large transverse phasespace. Downstream of the buncher the muons are cooled transversely within a $120 \mathrm{~m}$ long ionization cooling channel. The phase-space occupied by the muon bunches will then fit with the acceptance of an acceleration system consisting of a linac that accelerates the muons to $3 \mathrm{GeV}$, and two recirculating linear accelerators (RLA's) that raise the muon energies to $50 \mathrm{GeV}$. The muons are then injected into a storage ring. The ring is tilted downwards at the desired angle $\left(13^{\circ}\right)$, has a circumference of $1800 \mathrm{~m}$, and has two $600 \mathrm{~m}$ long straight sections. One third of the injected muons will decay in the downward pointing straight section. A detailed simulation of the Study I design shows that this scheme will produce about $6 \times 10^{19}$ muon decays per operational year (defined as $2 \times 10^{7}$ seconds) in the downward pointing straightsection, which is a factor of 3 less than the initial design goal for the study. The resulting beam intensity would be sufficient for a socalled "entry-level" Neutrino Factory, but insufficient for a "high-performance" machine.

Study II was initiated by the BNL Di- 


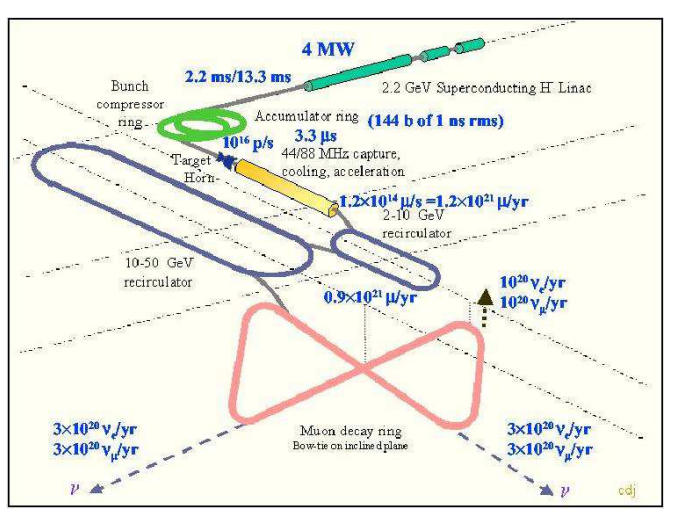

Figure 2. European Neutrino Factory schematic [12].

rectorate, and built upon the work done during Study I. The Study II was effort focussed on improving the Neutrino Factory design to achieve higher beam intensities. The main improvements came from using (i) a $4 \mathrm{MW}$ proton source, (ii) a liquid $\mathrm{Hg}$ target, (iii) an improved induction linac design, and (iv) an improved cooling channel design. Detailed simulations of the Study II design predicted $2 \times 10^{20}$ muon decays per operational year (defined as $1 \times 10^{7}$ seconds) in the downward pointing straight-section, thus achieving the initial goal.

\subsection{European Scheme}

The European Neutrino Factory design 12 has many similarities to the US design. However, the European studies have explored alternative technology choices for several key subsystems. A schematic is shown in Fig. 2. The European studies have focused on a design in which: (i) The proton driver consists of a $2.2 \mathrm{GeV}$ super-conducting linac (rather than a higher energy synchrotron), followed by an accumulator ring designed to produce short proton pulses 13 . The beam power is $4 \mathrm{MW}$. (ii) In addition to a liquid metal jet, a target consisting of water cooled Ta spheres is also being considered. (iii) The charged pions are focussed using a magnetic horn (rather than a high-field solenoid) with a $4 \mathrm{~cm}$ waist ra-

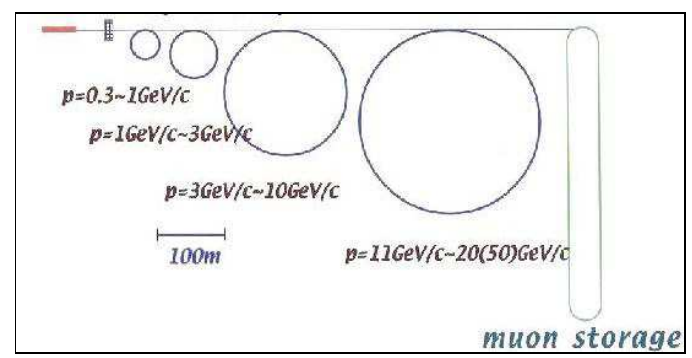

Figure 3. Japanese Neutrino Factory schematic [8].

dius, and a peak current of $300 \mathrm{kA}$. (iv) After a $30 \mathrm{~m}$ drift, the muon energy spread is reduced using $44 \mathrm{MHz}$ RF cavities (rather than induction an linac). (v) The ionization cooling channel (described later) uses $44 \mathrm{MHz}$ and $88 \mathrm{MHz}$ RF cavities. These are lower frequency cavities than employed in the US scheme. (vi) A bowtie-shaped storage ring (rather than a simple race-track design) has been considered for the final muon ring.

Simulations of the European design predict that the resulting neutrino beams will have intensities comparable to the corresponding beams from the US design. A comprehensive design study at the level of those in the US is not yet complete. It seems plausible that, at the end of the day, the optimal European/US-type Neutrino Factory design will inherit some of the technical choices being explored in the European studies, and some of those explored in the US studies.

\subsection{Japanese Scheme}

The Japanese Neutrino Factory scheme is shown in Fig. 3. The front end of the design consists of a $50 \mathrm{GeV}$ proton synchrotron providing a $4 \mathrm{MW}$ beam (corresponding to an upgraded JHF complex) incident on a target within a $12 \mathrm{~T}$ solenoid. This is followed by a large acceptance pion decay channel. However, instead of manipulating and cooling the phase space occupied by the muons exiting the decay channel, very large acceptance FFAG (Fixed Field Alternating 
Gradient) accelerators are used to raise the beam energy before injecting into a storage ring with long straight sections. The predicted neutrino yield from this scheme seems to be comparable to the corresponding predicted yields from the US/European designs, although more detailed simulations will be needed to confirm this.

Although the Japanese Neutrino Factory scheme might benefit from the addition of some muon cooling, in principle the use of FFAGs evades the need to cool the muons before injecting them into an accelerator. This simplification comes at the price of a more challenging accelerator, requiring complicated large aperture magnets and broadband low frequency high gradient RF cavities. Modern design tools have made practical the task of designing the FFAG magnets, and a small_proof-of-principle (POP) FFAG accelerator 14 has been built and successfully operated. A second test FFAG, designed to accelerate protons to $150 \mathrm{MeV}$, is under construction. Furthermore, with some US participation, an $R \& D$ program is underway in Japan to develop the required cavities. It is too early to conclude whether the promising Japanese Neutrino Factory scheme will lead to a more cost effective solution than the European/US-type designs. It may even be that the optimum solution consists of a combination of the two concepts, with some phase space manipulation and cooling, but using large acceptance FFAGs for the acceleration.

\section{Pion production \& Target R\&D}

To produce a sufficient number of useful $\pi^{ \pm}$, and hence a sufficient number of muons, all Neutrino Factory schemes begin with a MWscale proton driver, a pion production target, and a collection system optimized to capture as many $\pi^{ \pm}$as possible. To establish confidence in the predicted $\pi^{ \pm}$fluxes downstream of the target several secondary particle pro-

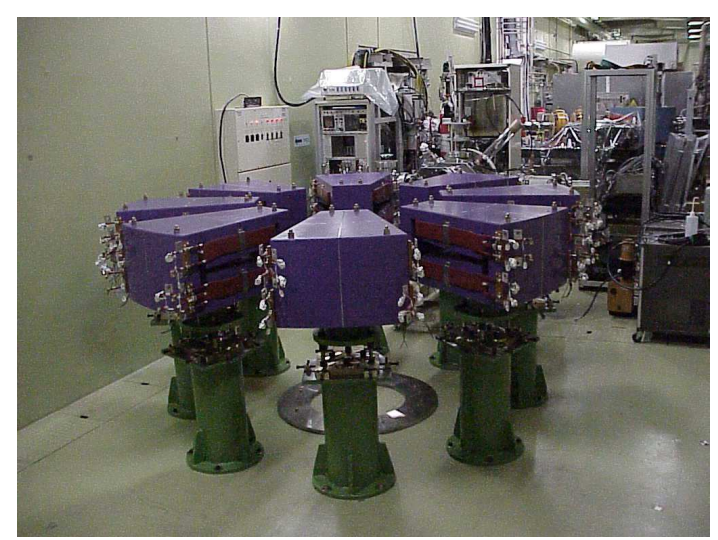

Figure 4. Proof of Principle (POP) FFAG accelerator [14].

duction experiments are underway 15, 16,17 . In addition, a target $R \& D$ program is being pursued to develop targets that can operate within MW-scale proton beams 18.

\subsection{Pion production experiments}

Figure 5 shows, as a function of the primary proton beam energy, predicted charged pion yields for $\pi^{+}$and $\pi^{-}$captured within a decay channel downstream of a Neutrino Factory target system. Over a broad interval of proton beam energies, at fixed beam power the yields are approximately independent of beam energy. Hence, a wide variety of multi$\mathrm{GeV}$ MW-scale proton drivers can be considered when designing a Neutrino Factory. The E910 experiment at BNL 15 has recently measured $\pi^{+}$and $\pi^{-}$yields for several targets and different incident proton beam energies. Some of the results are shown in Fig. 6 . Note that (i) the measurements are in fair agreement with MARS calculations 15, and (ii) the pion yields peak in the region 300 - $500 \mathrm{MeV} / \mathrm{c}$. Hence, Neutrino Factory designs tend to have $\pi^{ \pm}$collection systems optimized to capture particles with momenta in this range. In the next few years we can anticipate further particle production measurements from the HARP experiment 16 at CERN, and the proposed P907 experiment 17 


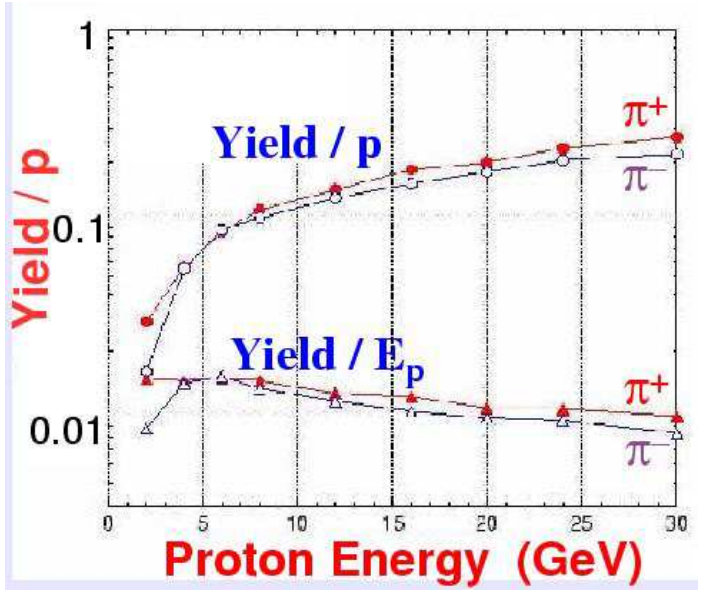

Figure 5. MARS predictions for the yield of pions captured within a Neutrino Factory pion decay channel, shown as a function of the primary proton beam energy [4]. More explicitly, the number of $\pi^{+}+\mu^{+}$ (filled symbols) and $\pi^{-}+\mu^{-}$(open symbols) within an energy interval $30 \mathrm{MeV}<E<230 \mathrm{MeV}$ is shown $9 \mathrm{~m}$ downstream of an $80 \mathrm{~cm}$ long $0.75 \mathrm{~cm}$ radius carbon target within a $20 \mathrm{~T}$ solenoid, and tilted $50 \mathrm{mrad}$ with respect to the solenoid axis. Triangles show the yields divided by the primary proton energy.

at Fermilab. This will enable the relevant $\pi^{ \pm}$ production measurements to be cross-checked and extended to cover the entire region of interest for Neutrino Factory designs.

\subsection{Target $R \mathscr{E} D$}

Entry-Level Neutrino Factories providing a few $\times 10^{19}$ useful muon decays per year require proton beams with beam powers of $\sim 1 \mathrm{MW}$, and short proton bunches, typically a few ns long. It is believed that carbon targets can operate with these beam parameters. This has been tested at BNL by the E951 Collaboration 19. Two different types of carbon rod were exposed to the AGS beam and strain gauge data taken. The beam induced longitudinal pressure waves and transverse reflections were both measured. The strains in the two types of rod differed by an order of magnitude, the most promising rod being made from an anisotropic carboncarbon composite.

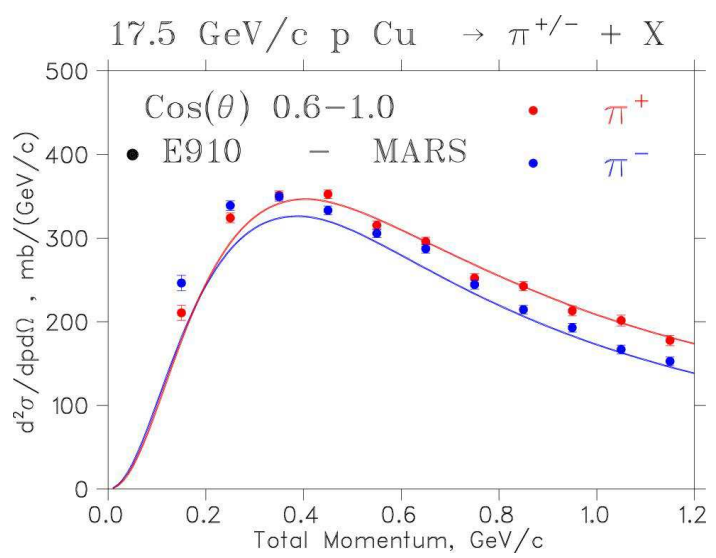

Figure 6. Pion production measurements from the BNL E910 experiment, compared with MARS predictions [15].

High performance Neutrino Factories providing $\mathrm{O}\left(10^{20}\right)$ useful muon decays per year require proton beams with beam powers of $\sim 4 \mathrm{MW}$. Targets must be developed to operate in these extreme conditions. Solid targets will melt unless very efficient cooling strategies can be developed. Rotating metal bands 20 and water cooled Ta spheres 21 are being considered, but the presently favored solution is to avoid problems with target melting and integrity by using a liquid $\mathrm{Hg}$ jet. In the US and Japanese schemes the target is in a high-field solenoid. Hence, the main $\mathrm{R} \& \mathrm{D}$ issues are (i) can a $\mathrm{Hg}$ jet be injected within a high-field solenoid without magneto-hydrodynamic effects disrupting the jet, (ii) after the jet has been destroyed by one proton pulse will it re-establish itself before the next pulse, and (iii) can the disrupted jet be safely contained within the target system.

To address these questions, $R \& D$ has begun at CERN and by the E951 collaboration at BNL. Photographs of a liquid $\mathrm{Hg}$ jet injected into a high-field solenoid at Grenoble are shown in Fig. 17. The presence of a $13 \mathrm{~T}$ solenoid field seems to damp surface tension waves, improving the characteristics of the jet. This is clearly good news. The E951 

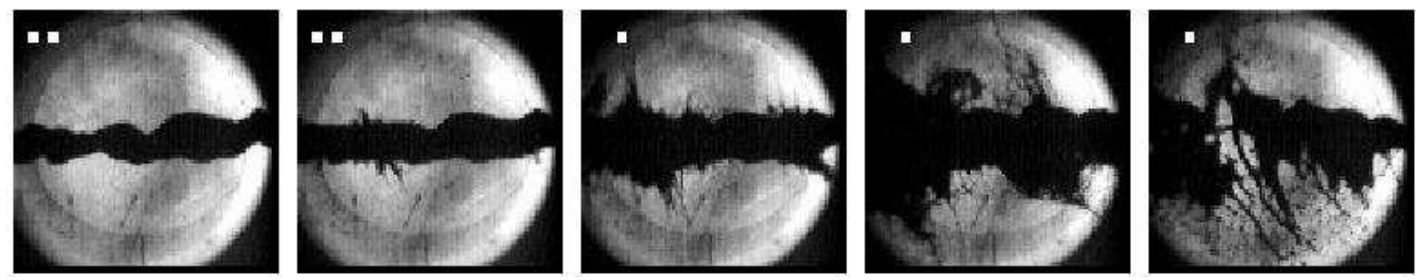

Figure 9. BNL E951 results [19]. The sequence of pictures shows the $\mathrm{Hg}$ jet at the time of impact $(\mathrm{t}=0)$ of the AGS beam, and at $\mathrm{t}=0.75 \mathrm{~ms}, 2 \mathrm{~ms}, 7 \mathrm{~ms}$, and $18 \mathrm{~ms}$. Time increases from left to right.

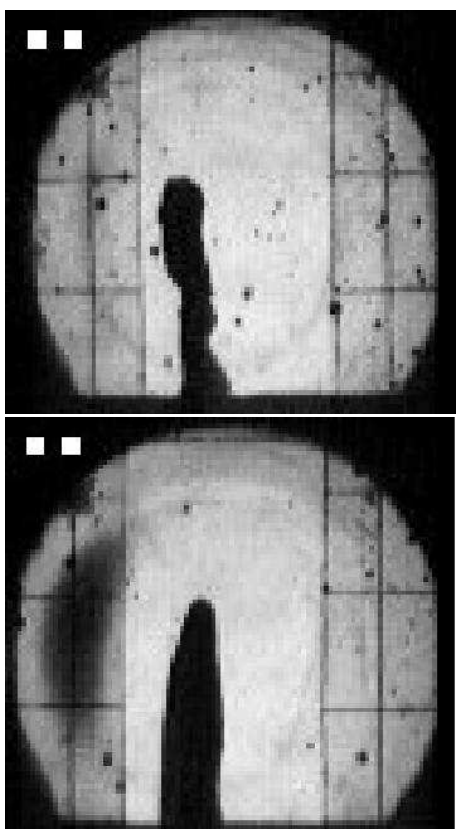

Figure 7. CERN-Grenoble tests in which a liquid $\mathrm{Hg}$ jet is injected into a solenoid providing 0 field (top picture) and $13 \mathrm{~T}$ (bottom picture) [13].

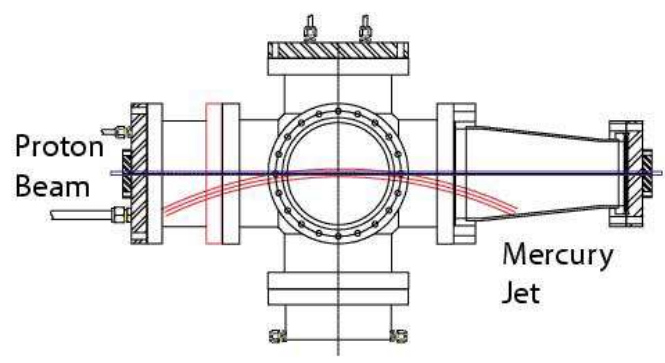

Figure 8. Schematic of the E951 system to test a Hg jet in the BNL AGS beam [19]. liquid $\mathrm{Hg}$ jet setup 19 in the BNL AGS beam is shown in Fig. 8. Figure 9 shows a sequence of pictures of the $1 \mathrm{~cm}$ diameter $\mathrm{Hg}$ jet taken by a high speed camera from the time of impact of $4 \times 10^{12}$ protons in a $150 \mathrm{~ns}$ long pulse from the $24 \mathrm{GeV}$ BNL AGS. Jet dispersal is delayed for about $40 \mu \mathrm{s}$, and it takes several $\mathrm{ms}$ before the jet, which has an initial velocity of $2.5 \mathrm{~m} / \mathrm{sec}$, is completely disrupted. The velocity of the out-flying $\mathrm{Hg}$ filaments, which appears to scale with beam intensity, has been measured to be $\sim 10 \mathrm{~m} / \mathrm{sec}$ for a deposited energy of $25 \mathrm{~J} / \mathrm{g}$. These velocities are fairly modest, implying that the disrupted jet can be easily contained within the target system. Furthermore, the Hg jet dispersal is mostly in the transverse direction, and after disruption it has been found that the jet quickly re-establishes itself.

Initial results with $\mathrm{Hg}$ jets are promising. However, high performance Neutrino Factories will require $\mathrm{Hg}$ jets with much higher velocities $(\sim 20 \mathrm{~m} / \mathrm{sec})$ to be developed and tested. The next steps in the E951 R\&D program will require beam tests with a factor of a few higher beam intensities, and finally beam tests in which the $\mathrm{Hg}$ jet is injected into a $20 \mathrm{~T}$ solenoid.

\section{Muon Cooling R\&D}

Before the muons can be accelerated the transverse phase-space they occupy must be reduced so that the muon beam fits within the acceptance of an accelerator. This means the muons must be "cooled" by at least a 


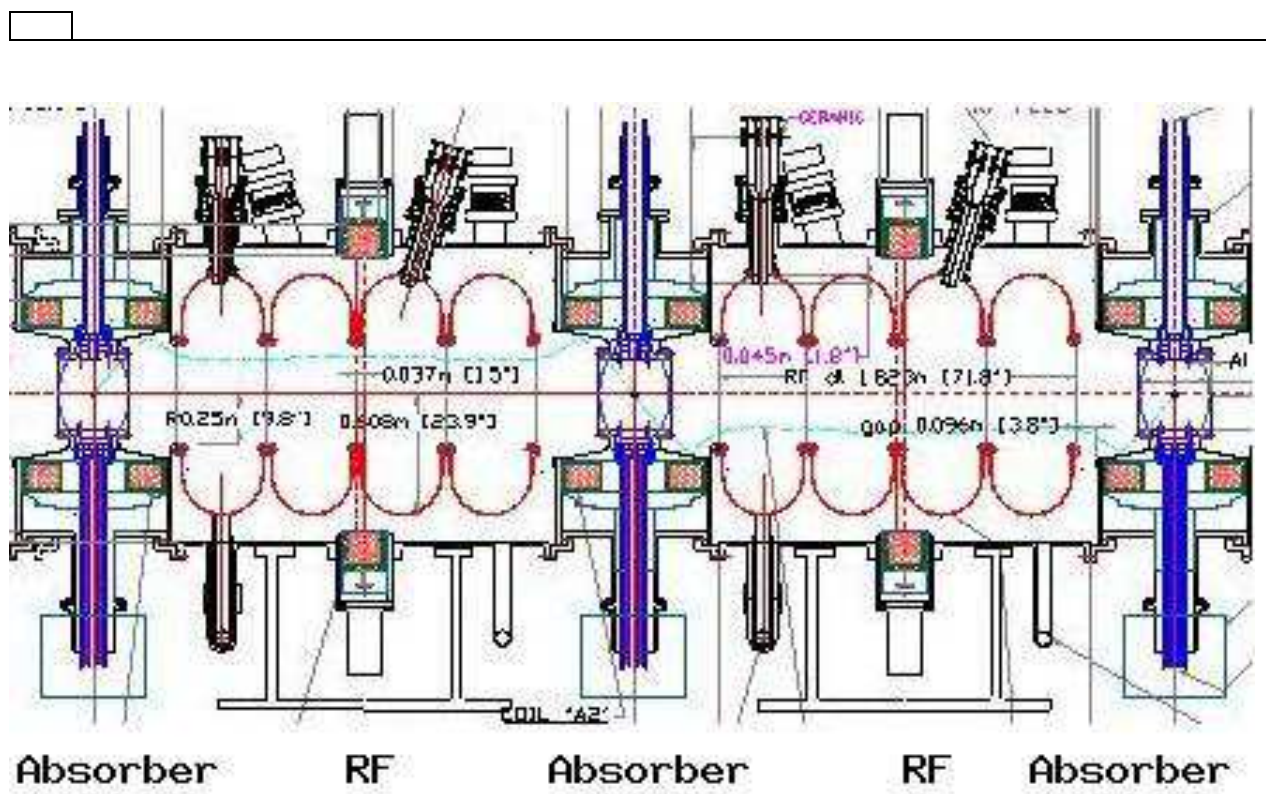

Figure 10. SFOFO cooling channel design [5]. A $5.5 \mathrm{~m}$ long section is shown, consisting of two $200 \mathrm{MHz}$ four-cell cavities interleaved with three liquid hydrogen absorbers.

factor of a few in each transverse plane, and this must be done fast, before the muons decay. Stochastic- and electron-cooling are too slow. It is proposed to use a new cooling technique, namely "ionization cooling" 10 .

In an ionization cooling channel the muons pass through an absorber in which they lose transverse- and longitudinalmomentum by $d E / d x$ losses. The longitudinal momentum is then replaced using an RF cavity, and the process is repeated many times, reducing the transverse momenta. This cooling process will compete with transverse heating due to Coulomb scattering. To minimize the effects of scattering we chose low $-\mathrm{Z}$ absorbers placed in the cooling channel lattice at positions of low $-\beta_{\perp}$ so that the typical radial focusing angle is large. If the focusing angle is much larger than the average scattering angle then scattering will not have much impact on the cooling process.

\subsection{US Cooling Channel Design}

Studies I and II used two simulation tools developed by the Neutrino Factory and Muon Collider Collaboration: (i) A specially de- veloped tracking code ICOOL 22, and (ii) A GEANT based program with accelerator components (e.g. RF cavities) implemented. Out of these design and simulation studies, two promising cooling channel designs have emerged:

(i) The "SFOFO" lattice in which the absorbers are placed at low $-\beta_{\perp}$ locations within high-field solenoids. The field rapidly decreases from a maximum to zero at the absorber center, and then increases to a maximum again with the axial field direction reversed. Figure 10 shows the design for a $5.5 \mathrm{~m}$ long section of the $\sim 100 \mathrm{~m}$ long cooling channel. The section shown has $30 \mathrm{~cm}$ long absorbers with a radius of $15 \mathrm{~cm}$, within a system of solenoids with a peak axial field of $3.5 \mathrm{~T}$. Towards the end of the cooling channel the maximum field is higher $(5 \mathrm{~T})$ and the lattice period shorter $(3.3 \mathrm{~m})$. The RF cavities operate at $201 \mathrm{MHz}$ and provide a peak gradient of $17 \mathrm{MV} / \mathrm{m}$. Detailed simulations predict that the SFOFO channel increases the number of muons within 
the accelerator acceptance by a factor of 3-5 (depending on whether a largeor very-large acceptance accelerator is used).

(ii) The "DFLIP" lattice in which the solenoid field remains constant over large sections of the channel, reversing direction only twice. In the early part of the channel the muons lose mechanical angular momentum until they are propagating parallel to the axis. After the first field flip the muons have, once again, mechanical angular momentum, and hence move along helical trajectories with Lamour centers along the solenoid axis. Further cooling removes the mechanical angular momentum, shrinking the beam size in the transverse directions. The field in the early part of the channel is $3 \mathrm{~T}$, increasing to $7 \mathrm{~T}$ for the last part. Detailed simulations show the performances of the DFLIP and SFOFO channels are comparable.

Earlier less detailed studies 1 have shown that a much larger cooling factor will be required for a muon collider. This will require an extended cooling channel, using higher frequency (e.g. $805 \mathrm{MHz}$ ) cavities, higher field solenoids, and possibly liquid lithium lenses 23.

\subsection{MUCOOL RED}

Muon cooling channel design and development is being pursued within the US by the MUCOOL collaboration 24. The mission of the MUCOOL collaboration includes benchtesting all cooling channel components, and eventually beam-testing a cooling channel section. The main component issues that must be addressed before a cooling channel can be built are (i) can sufficiently high gradient RF cavities be built and operated in the appropriate magnetic field and radiation environment, (ii) can liquid hydrogen absorbers with thin enough windows be built

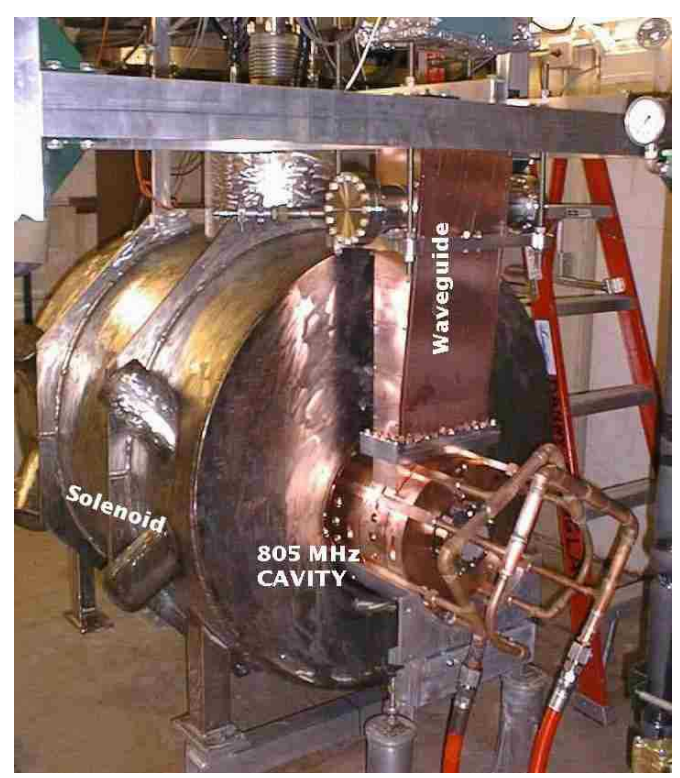

Figure 11. High power $805 \mathrm{MHz}$ cavity test in the MUCOOL Lab G test area, Fermilab [25].

so that the $d E / d x$ heating can be safely removed, and (iii) can the lattice solenoids be built to tolerance and be affordable? The MUCOOL collaboration has embarked on a design-, prototyping-, and testing-program that addresses these questions. This R\&D is expected to proceed over the next 3 years.

\section{$805 \mathrm{MHz}$ RF Tests}

Early design work for a Muon Collider resulted in a Muon Collider cooling channel design that required $805 \mathrm{MHz}$ cavities operating in a $5 \mathrm{~T}$ solenoid, with the cavities providing a peak gradient on axis of $\sim 30 \mathrm{MV} / \mathrm{m}$. This deep potential well is needed to keep the muons bunched as they propagate down the channel. This requirement led to two cavity concepts: (a) an open cell design, and (b) a design in which the penetrating nature of the muons is exploited by closing the RF aperture with a thin conducting Be window (at fixed peak power this doubles the gradient on axis).

The MUCOOL collaboration has pur- 


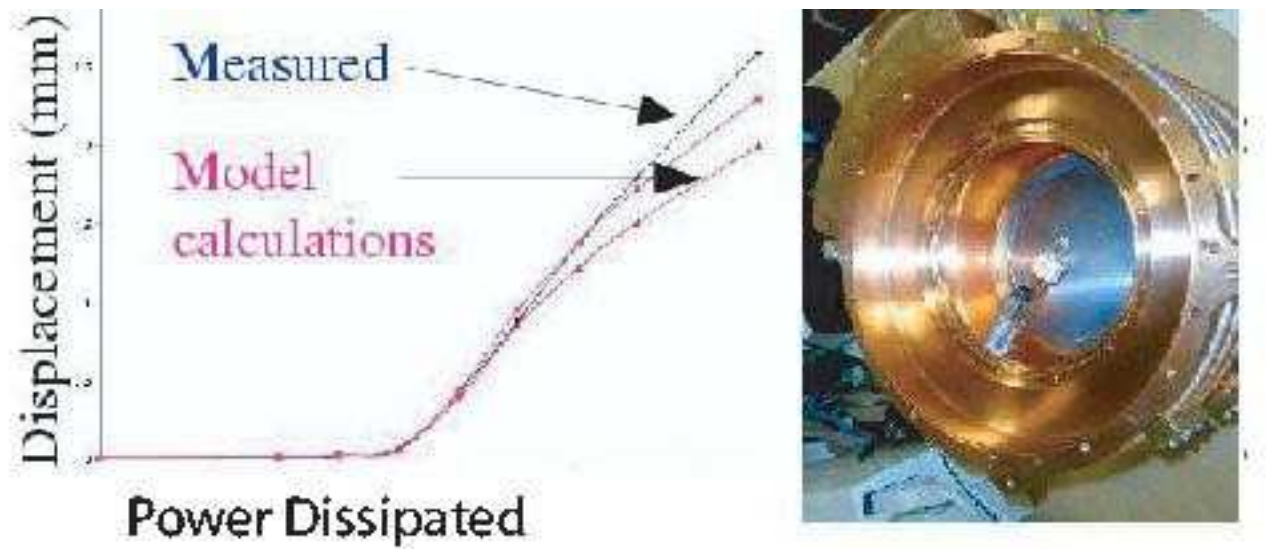

Figure 12. Measurements of the deflection of a Be foil in an $805 \mathrm{MHz}$ cavity at LBNL [26]. The measured deflections are shown as a function of the heat dissipated in the foil, and are compared with predictions from two finite element analysis calculations.

sued an aggressive $805 \mathrm{MHz}$ cavity development program, which is now advanced. A $12 \mathrm{MW}$ high power test facility has been built and operated at Fermilab (Lab G). The Lab G facility enables $805 \mathrm{MHz}$ cavities to be tested within a $5 \mathrm{~T}$ solenoid. The main results to date are: (i) An open cell cavity suitable for a muon cooling channel has been designed, an aluminum model built and measured, and a prototype copper cavity built, tuned, and successfully tested at full power in the Lab G facility (Fig. 11). Dark current produced by the cavity has been identified as an important R\&D issue 25 . (ii) A Be foil cavity has been designed at LBNL, a low power test cavity built and measured, and foil deflection studies made 22 to ensure the cavity does not detune when the foil is subject to RF heating. The foil deflection is reasonably well understood for small displacements (Fig. 12). A high power copper cavity with Be-foil windows is under construction at LBNL and the University of Mississippi, and will be tested at Lab $\mathrm{G}$ when ready.

\section{$201 \mathrm{MHz}$ Cavity Development}

The cooling channel designs developed for the US Neutrino Factory studies require $201 \mathrm{MHz}$ $\mathrm{RF}$ cavities providing a gradient on axis of $\sim 17 \mathrm{MV} / \mathrm{m}$. Preliminary cavity designs have been made. There are two concepts, both of which close the cavity aperture. The options are to use (a) a thin Be foil, exploiting the work done for the $805 \mathrm{MHz}$ cavity, or (b) use a grid of hollow conducting tubes. Preliminary mechanical tests for both the grid and foil concepts are planned, and should proceed during the next few months. A $201 \mathrm{MHz}$ prototype cavity will then be constructed, and should be ready for high power tests in about 2 years.

\section{Absorber Development}

The cooling channel liquid hydrogen absorbers must have very thin windows to minimize multiple scattering, and must tolerate heating of $\mathrm{O}(100 \mathrm{~W})$ from the ionization energy deposited by the traversing muons. Absorber parameters for the Neutrino Factory study II cooling channel design are listed in Table 1 .

To adequately remove the heat from the absorbers requires transverse mixing of the liquid hydrogen. There are two design concepts that are being pursued 27: (i) Forced flow design. The $\mathrm{LH}_{2}$ is injected into the absorber volume through nozzles, and cooled using an external loop and heat exchanger. 
Table 1. $\mathrm{LH}_{2}$ absorber parameters in Neutrino Factory design study II [5].

\begin{tabular}{l|cccccc}
\hline \hline Absorbers & $\begin{array}{c}\text { Length } \\
(\mathrm{cm})\end{array}$ & $\begin{array}{c}\text { Radius } \\
(\mathrm{cm})\end{array}$ & $\begin{array}{c}\text { Number } \\
\text { Needed }\end{array}$ & $\begin{array}{c}\text { Heat }(\mathrm{kW}) \\
\text { Deposited }\end{array}$ & $\begin{array}{c}\text { Window Thick } \\
\text {-ness }(\mu \mathrm{m})\end{array}$ & $\begin{array}{c}\text { Max. Pres- } \\
\text { sure }(\mathrm{atm})\end{array}$ \\
\hline Early & 35 & 18 & 16 & $\sim 0.3$ & 360 & 1.2 \\
Late & 21 & 11 & 36 & $\sim 0.1$ & 220 & 1.2 \\
\hline \hline
\end{tabular}
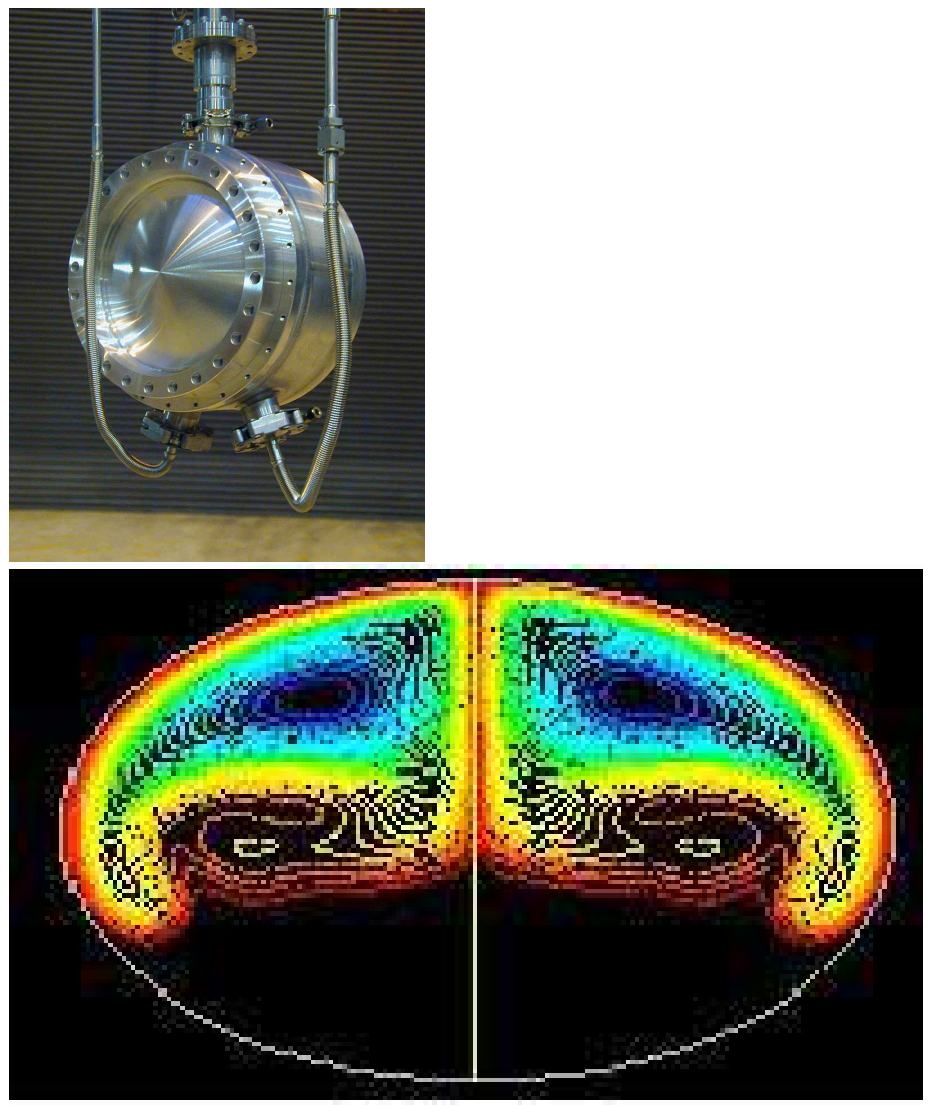

Figure 13. KEK prototype absorber. The liquid hydrogen is to be mixed by convection and cooled with a local heat exchanger. A simulation of the convection, performed at IIT, is shown on the right. 
(ii) Convection design. Convection is driven by a heater at the bottom of the absorber volume, and heat removed by a heat exchanger on the outer surface of the absorber. A forced flow absorber prototype is being designed at the Illinois Institute of Technology (IIT) and will be constructed in the coming year. A convection prototype, designed by IIT, KEK, and the University of Osaka, is being constructed in Japan (Fig. 13). Both absorbers will be tested at Fermilab when complete.

A first prototype $15 \mathrm{~cm}$ radius aluminum absorber window has been made at the University of Mississippi on a CNC milling machine and lathe. The window has a central thickness of $130 \mu \mathrm{m}$. The window thickness and profile were measured at FNAL and found to be within $5 \%$ of the nominal envelope. This verifies the manufacturing procedure. The window has been tested 27 under pressure in a setup at Northern Illinois University in which it was mounted on a backplate and water injected between window and plate. Strain gauge and photogrammetric measurements were made as a function of pressure, and the results compared with FEA predictions. The onset of inelastic deformation was predicted at $29 \mathrm{psig}$, a pinhole leak appeared at $31 \mathrm{psig}$, and rupture occurred at 44 psig. The windows required for a cooling channel absorber can be about twice as thick as the first prototype window. The results to date are therefore encouraging. Further window studies and tests are proceeding.

\subsection{European Cooling REDD Program}

The European cooling channel design 28 is similar in concept to the US design, but is based on $44 \mathrm{MHz}$ and $88 \mathrm{MHz}$ cavities 29 rather than $201 \mathrm{MHz}$ cavities. To minimize the radii of the solenoids used to confine the muons within the channel, the cavities have been designed to wrap around the solenoids. A full engineering design of this concept will be required to understand its feasibility. The

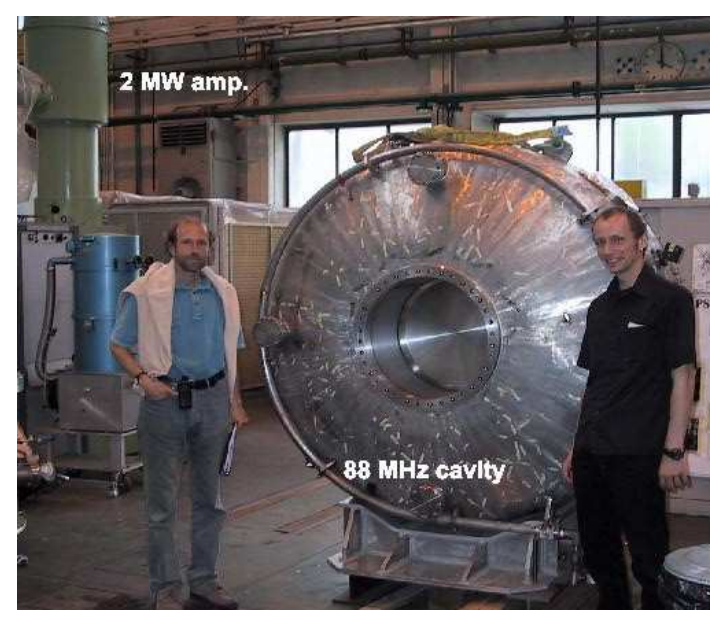

Figure 15. CERN $88 \mathrm{MHz}$ cavity [29] to be prepared for high power tests.

initial transverse cooling is performed using $44 \mathrm{MHz}$ cavities with four $1 \mathrm{~m}$ long RF cells between each $24 \mathrm{~cm}$ long $\mathrm{LH}_{2}$ absorber. The beam is then accelerated from $200 \mathrm{MeV}$ to $300 \mathrm{MeV}$, and the cooling is continued using $88 \mathrm{MHz}$ cavities with eight $0.5 \mathrm{~m}$ long cells between each $40 \mathrm{~cm}$ long $\mathrm{LH}_{2}$ absorber. The channel parameters are summarized in Table 2. Simulations of the channel performance with detailed field-maps have not yet been made. However, simulations using simpler field maps yield promising results: the effect of the channel is to increase the number of muons within the acceptance of the subsequent accelerating system by a factor of about 20 . Whether or not the predicted increased yield is significantly degraded when full simulations are performed remains to be seen. In the meantime, a prototype $88 \mathrm{MHz}$ cavity is being prepared at CERN (Fig. 15) for high power tests within the coming year.

\subsection{Cooling Experiments}

A sequence of muon cooling-related experiments is being planned. The first, the MUSCAT experiment 30, is already under way at TRIUMF. The second, the MUCOOL Com- 


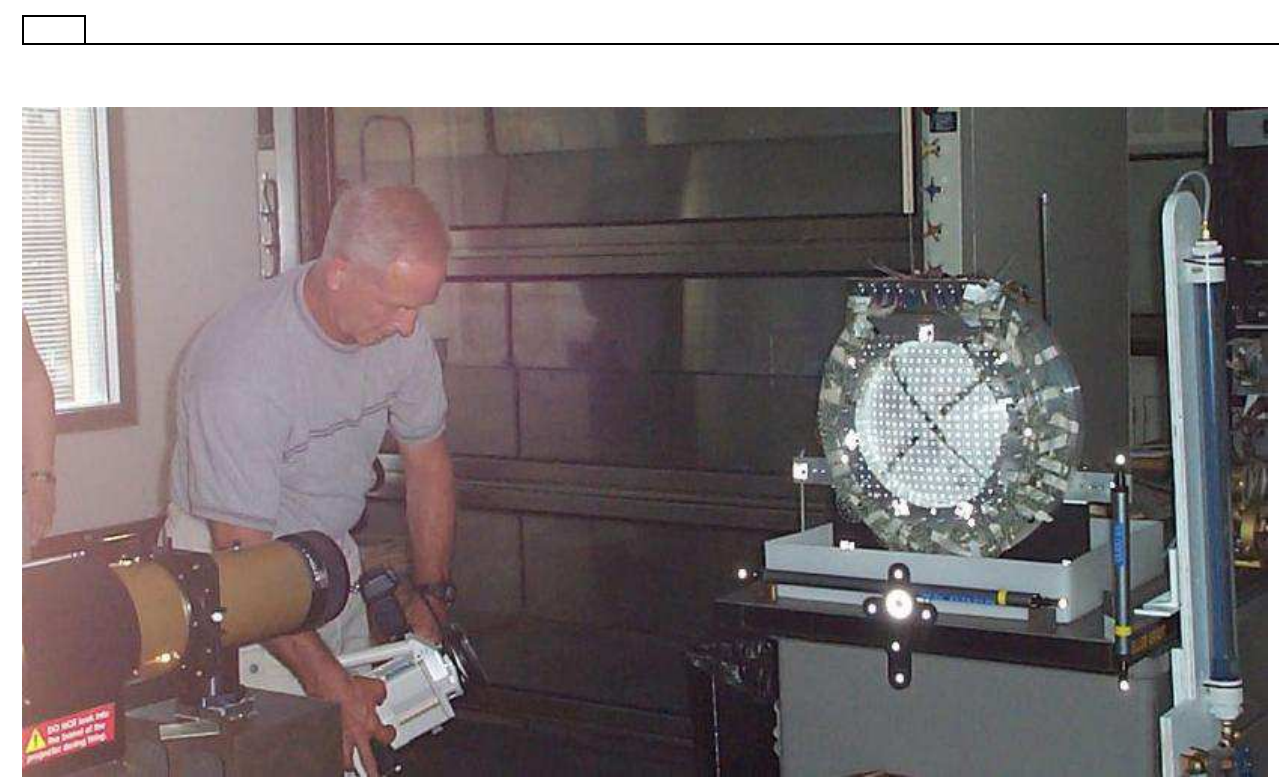

Figure 14. Absorber window test, showing an array of dots projected onto the window for photogrammetric measurements of its shape as it deforms under pressure [27].

Table 2. CERN cooling channel design parameters [28].

\begin{tabular}{l|cc}
\hline \hline & Channel 1 & Channel 2 \\
\hline Length & $46 \mathrm{~m}$ & $112 \mathrm{~m}$ \\
Diameter & $60 \mathrm{~cm}$ & $30 \mathrm{~cm}$ \\
Sol. Field & $2.0 \mathrm{~T}$ & $2.6 \mathrm{~T}$ \\
RF Freq. & $44 \mathrm{MHz}$ & $88 \mathrm{MHz}$ \\
RF Gradient & $2 \mathrm{MV} / \mathrm{m}$ & $4 \mathrm{MV} / \mathrm{m}$ \\
Beam Energy & $200 \mathrm{MeV}$ & $300 \mathrm{MeV}$ \\
\hline \hline
\end{tabular}

ponent Test Experiment, is under construction at the Fermilab Linac. The third, an International Cooling Experiment 31, is in the planning stage. The fourth, an eventual String Test Experiment, will be planned in the future.

\section{MUSCAT}

The goal of the MUSCAT experiment at TRI$\mathrm{UMF}$ is the precise measurement of low energy $(130,150$, and $180 \mathrm{MeV} / \mathrm{c})$ muon scattering in a variety of materials that might be found within a cooling channel. In a second phase, the experiment will also measure straggling. Scattering measurements for Li,
$\mathrm{Be}, \mathrm{C}, \mathrm{Al}, \mathrm{CH}_{2}$, and $\mathrm{Fe}$ have already been made. Preliminary results seem to be in good agreement with expectations 30 . Further analysis is in progress. Measurements with $\mathrm{LH}_{2}$ are expected in the future.

\section{MUCOOL Component Test Experiment}

A MUCOOL test area located at the end of the Fermilab $400 \mathrm{MeV}$ Linac was proposed in the Fall of 2000, and is currently under construction. The project is being pursued in two phases. In Phase 1 a $\mathrm{LH}_{2}$ absorber test facility is being built, which will enable the first prototype absorbers to be filled. In Phase 2 a linac beam will be brought to the absorber area, and the $5 \mathrm{~T}$ solenoid will be moved from Lab $\mathrm{G}$ so that the absorber can be tested in a magnet whilst exposed to a proton beam. The beam intensity and spot size will be designed to mimic the total ionization energy deposition and profile that corresponds to the passage of $10^{12}-10^{13}$ muons propagating through a cooling channel. In addition, $201 \mathrm{MHz}$ RF power will be piped to the test area from a nearby test-stand, enabling high-power tests to be made of a proto- 
type $201 \mathrm{MHz}$ cooling channel cavity exposed to the proton beam.

\section{International Cooling Experiment}

A Europe-Japan-US International Cooling Experiment is currently being planned 31 . The goals are to (i) place a cooling channel section in a muon beam, and (ii) demonstrate our ability to precisely simulate the passage of muons confined within a periodic lattice as they pass through $\mathrm{LH}_{2}$ absorbers and highgradient RF cavities. In the envisioned experiment muons are measured one at a time at the input and output of the cooling section, and the precise response of the muons to the cooling section is determined. The main challenge to the design of this type of experiment arises from the prolific X-ray and dark current environment created by the RF cavities. This is currently under study at Lab G and elsewhere. If it is found that single particle detectors can function in this hostile environment, we anticipate a proposal being submitted sometime in 2002.

\section{Muon Acceleration and Storage}

The acceleration system has been identified as one of the cost drivers for a Neutrino Factory. In the US and European schemes the main acceleration systems use SC cavities. The US scheme, for example, uses $201 \mathrm{MHz}$ SCRF delivering gradients of $15 \mathrm{MV} / \mathrm{m}$ with $\mathrm{Q} \sim 5 \times 10^{9}$. Although higher frequency $\mathrm{SCRF}$ cavities are no longer novel, $201 \mathrm{MHz}$ is a relatively low frequency and the cavities are therefore large. The associated $\mathrm{R} \& \mathrm{D}$ issues are related to microphonics, fabrication and cleaning techniques and, because of the large stored energy, quench protection. Furthermore, the cavities must tolerate whatever stray magnetic fields they see within the accelerating lattice. To address these issues a $201 \mathrm{MHz}$ SC cavity is being constructed at CERN and sent to Cornell for high-power testing.
No major R\&D issues have been identified for the final muon storage ring. Building a ring tilted at a large angle raises interesting, but not insurmountable, construction challenges $\mathrm{t}^{\mathrm{a}}$.

\section{Muon Collider Issues}

A Neutrino Factory, although motivated by its own physics program, would also provide a solid step in developing the technology that would be required to eventually build a Muon Collider. However, additional issues must be addressed before a Muon Collider could be proposed. In particular:

(i) Muon Cooling: The 6-D cooling factor required for a Muon Collider is $\mathrm{O}\left(10^{6}\right)$ compared with the more modest factor of $\mathrm{O}(100)$ for a Neutrino Factory. Muon cooling for a Muon Collider will require additional technology. In particular, present Muon Collider muon cooling schemes require the longitudinal phase-space to be reduced using "emittance exchange" in which some of the reduction in the transverse phase-space is traded for a reduction in the muon energy spread. Although progress towards a viable emittance exchange scheme has been made over the last two years, a convincing design has not yet emerged. In addition, to obtain the final transverse emittances required for a Muon Collider will require a cooling channel with stronger radial focusing than is likely to be achieved with affordable high-field solenoids. A new technology (liquid lithium lenses 23 , optical stochastic cooling $32, \ldots$ ) is required.

(ii) Cost-effective acceleration: Acceleration is a cost driver for a Neutrino Factory, which requires muons accelerated to 20 $50 \mathrm{GeV}$. If a multi-TeV Muon Collider is to be affordable an efficient cost-effective acceleration system must be developed. 
(iii) Single muon bunches: The Neutrino Factory does not require the muons to be packaged into a small number of bunches. However, to maximize the luminosity, the muons for a Muon Collider should be packaged into one $\mu^{+}$and one $\mu^{-}$bunch per cycle. This will require a more challenging bunching scheme and raises additional issues associated with having more intense muon bunches (e.g. space charge effects).

(iv) Detector backgrounds: Decaying muons within the Muon Collider ring create an intense flux of energetic electrons in the neighborhood of the detector. This has been studied in detailed 33, and elaborate shielding strategies have been shown to reduce the backgrounds down to levels that appear to be acceptable.

Assuming that solutions can be found on paper for these Muon Collider design challenges, I believe it will take many extra years of R\&D to develop the additional technology required for a Multi-TeV Muon Collider. However, the best way to eventually build a cost-effective multi-TeV lepton collider is far from clear, and R\&D addressing Muon Collider issues is worthy of a significant investment by the community.

\section{Summary and Prospects}

Muon sources capable of delivering a millimole of muons per year seem feasible, and would enable Neutrino Factories, and perhaps eventually Muon Colliders, to be built. Neutrino Factory designs have been developed in Europe, Japan, and the US. Three promising schemes are being studied, and a healthy R\&D program is underway to develop the required technologies. The most challenging R\&D questions are associated with targets for MW-scale proton beams, and the development of an ionization cooling channel. Additional challenges must be overcome before a Muon Collider can be proposed.

With the present level of support, we can expect much progress in Neutrino Factory R\&D over the next few years. However, the future level of support is uncertain, and I believe a significant increase (factor of two ?) in the R\&D support will be needed, sustained over a handful of years, if we are ever to arrive at a "Technical Design Report". In addition, there must be good international collaboration to enable the most promising design to be eventually chosen, and pursued. There is already a healthy dialogue between the European, Japanese, and US R\&D teams, and some cross-participation in the various $R \& D$ programs. This international collaboration would greatly benefit from an increase in support to enable, for example, an international ionization cooling experiment.

Finally, it should be noted that Neutrino Factory R\&D is being pursued by engineers, accelerator physicists, and particle physicists from Laboratories and Universities in Europe, Japan and the US. There are a broad range of interesting sub-projects to be pursued, and with adequate support, the prospects seem bright.

\section{Acknowledgments}

This talk is based upon the work of many people. I am particularly indebted to material provided to me from my colleagues in Europe and Japan, most notably B. Autin, H. Haseroth, Y. Kuno, and Y. Mori. In addition I am indebted to all the members of the US Neutrino Factory and Muon Collider Collaboration, and those outside of the collaboration who have participated in the two US Neutrino Factory studies. My own humble contribution to the work was supported at the Fermi National Accelerator Laboratory, which is operated by Universities Research Association, under contract No. DE-AC02$76 \mathrm{CH} 03000$ with the U.S. Department of En- 
ergy.

\section{References}

1. S. Geer, Phys. Rev. D57, 6989 (1998).

2. G.I. Budker, AIP Conf. Proc. 352 (AIP, New York, 1996) p.4; A.N. Skrinsky, AIP Conf. Proc. 352 (AIP, New York, 1996) p.6; C.M. Ankenbrandt et al. (Muon Collider Collabration), " $\mu^{+} \mu^{-}$Collider: A Feasibility Study", Snowmass 96, BNL-52505; ibid. Phys. Rev. ST Accel. Beams 2, 081001 (1999).

3. B. Autin, A. Blondel, J. Ellis (editors), CERN yellow report 99-02., C. Albright et al., (Eds. S. Geer and H. Schellman), "Physics at a Neutrino Factory", Report to the Fermilab Directorate, May 10, 2000, hep-ex/0008064. see also the proceedings of NUFACT99 (Lyon, France), NUFACT00 (Monterey, US), and NUFACT01 (Tsukuba, Japan). There are many other neutrino factory physics papers. Amongst the most cited are: A. De Rujula, M.B. Gavela, P. Hernandez, Nucl.Phys.B547:21-38,1999; V. Barger, S. Geer, R. Raja, K. Whisnant, Phys.Rev.D62:013004,2000., and A. Cervera et al., Nucl.Phys.B579:1755,2000, Erratum-ibid.B593:731,2001.

4. T. Anderson et al. (Eds: N. Holtkamp and D. Finley), "A Feasibility Study of a Neutrino Source Based on a Muon Storage Ring", FERMILAB-Pub-00/108-E (2000).

5. M. Goodman et al. (Eds: S. Ozaki, R. Palmer, M. Zisman, J. Gallardo), "Feasibility Study II of a Muon-Based Neutrino Source", BNL-52623, June 2001.

6. NufactJ Working Group (Eds. Y. Kuno, Y. Mori), "A Feasibility Study of a Neutrino Factory in Japan", see http:// www-prism.kek.jp/ nufactj/ index.html

7. R. Garoby and B. Autin, presentions at the Neutrino Factory Work- shop NUFACT01, Tsukuba, Ibaraki, Japan, May 24-30, 2001; B. Autin, "European R\&D for Neutrino Factory", CERN Neutrino Factory Note 101, see http://molat.home.cern.ch/molat/ neutrino/nfnotes.html

8. S. Machida, presented at the Neutrino Factory Workshop NUFACT01, Tsukuba, Ibaraki, Japan, May 24-30, 2001.

9. R. Raja et al; "The Program in Muon Physics: Superbeams, Cold Muon Beams, Neutrino Factory and Muon Collider", Contributed to APS / DPF / DPB Summer Study on the Future of Particle Physics (Snowmass 2001), Snowmass, Colorado, 30 Jun - 21 Jul 2001; hep-ex/0108041

10. A.N. Skrinsky and V.V. Parkhomchuk, Sov. J. of Nuclear Physics, 12, 3 (1981); D. Neuffer, Particle Accelerators 14, 75 (1983).

11. R. Alber et al. (Eds. W. Chou, A. Ankenbrandt, and E. Malamud), "The Proton Driver Design Study", FERMILAB-TM-2136, Dec 2000.

12. H. Haseroth (CERN). CERN-PS-2000064-PP.

13. B. Autin (CERN). CERN-PS-2000-067PP, Workshop on High Intensity Muon Sources, Tsukuba, Japan, 1-4 Dec 1999; B. Autin et al., CERN-PS-2000-011-AE, Jun 2000. 4pp., 7th European Particle Accelerator Conference, Vienna, Austria, 26-30 Jun 2000. B. Autin et al., CERN-2000-012, Dec 2000. 89pp.

14. Y. Mori, Nuclear Instruments and Methods in Physics Research Section A, Vol. 451 (1) (2000) p. 300.

15. I. Chemakin et al. (E910 Collab.), "Inclusive Soft Pion Production from 12.3 and $17.5 \mathrm{GeV} / \mathrm{c}$ Protons on $\mathrm{Be}, \mathrm{Cu}$, and Au", nucl-ex/0108007.

16. Proposal to Study Hadron Production for the Neutrino Factory and for the Atmospheric Neutrino Flux", 
CERN-SPSC/99-35, SPSC/P315, 15 November, 1999 HARP Web Page: http://harp.web.cern.ch/harp/

17. P907: "Proposal to measure particle production in the Meson area using Main Injector primary and secondary beams", Y.Fisyak et al., R.Raja (spokesperson), http://ppd.fnal.gov/experiments/ e907/e907.htm

18. J. Alessi et al. (E951 Collab.), "Proposal: An R\&D Program for Targetry and Capture at a Muon Collider Source", September 28, 1998.

19. A. Hassenein et al., "An R\&D Program for Targetry and Capture at a Neutrino Factory and Muon Collider Source", Proc. Neutrino Factory Workshop NUFACT01, Tsukuba, Ibaraki, Japan, May 24-30, 2001.

20. B. King et al; "A Rotating Inconel Band Target for Pion Production at a Neutrino Factory", MUCOOL Note 199, see http://www-mucool.fnal.gov/htbin/ mcnote1LinePrint

21. P. Sievers, "A Stationary Target for the CERN Neutrino Factory", CERN Neutrino Factory Note 65. See http: // molat.home.cern.ch/molat/neutrino/nfnotes.

22. R. Fernow, "Fortran Program to Simulate Ionization Cooling", unpublished.

23. B. Bayanov et al., "Liquid Lithium Lens for Fermilab Antiproton Source", Budker INP 98-23; B. Bayanov et al., "Design and Construction Technology for a 15 cm Long Liquid Lithium Lens", Budker Institute of Nuclear Physics, 1998.

24. The MUCOOL Web Page can be found at http://www.fnal.gov / projects /muon_ collider / cool/ cool.html

25. J. Norem et al., "Preliminary Lab G Dark Current Results", MUCOOL Note 226 at http://www-mucool.fnal.gov/htbin / monote1LinePrint

26. N. Hartman, Derun Li, and J. Corlett, "Thin Beryllium Windows - Analysis and Design Status", MUCOOL Note
180, see http://www-mucool.fnal.gov/ htbin/ mcnote1LinePrint

27. D. Kaplan et al., "Progress in Absorber R\&D for Muon Cooling", physics 0108027; D. Kaplan et al., "Progress in Absorber R\&D 2: Windows", physics, 0108028 .

28. A. Lombardi, "A 40-80 MHz System for Phase Rotation and Cooling", CERN Neutrino Factory Notes 20 and 34, see http://molat.home.cern.ch/molat/ neutrino/nfnotes.html

29. R. Garoby and F. Gerigk, "Cavity Design for the CERN Muon Cooling Channel", CERN Neutrino Factory Note 87, see http://molat.home.cern.ch/molat/ neutrino/nfnotes.html

30. B. Autin, talk given at NUFACT01.

31. K. Hanke, talk given at NUFACT01, and http://hep04.phys.iit.edu/cooldemo/.

The cooling experiment was first proposed in the Fermilab P907 proposal: C.Ankenbrandt et al., "Proposal: Ionization Cooling R\&D Program for a High Luminosity Muon Collider", April 15, 1998 (see http: // www.fnal.gov/projects/muon _ colhtml lider/cool/ proposal/ proposal.html).

32. A. Zholents, M. Zolotorev, and W. Wan; Phys. Rev. Special Topics - Accelerators and Beams, 4, 031001 (2001).

33. Muon Collider Collaboration, in Proceedings of the 1996 Snowmass Study, Chapter 9 of the Muon Collider Feasibility Study. 